\title{
A Unified Phenomenological Model for Tensile and Compressive Response of Polymeric Foams
}

\section{Andrew W. Richards \\ Mechanical Engineering, \\ California Institute of Technology, \\ Pasadena, CA 91125}

\author{
Ghatu Subhash ${ }^{1}$ \\ Mechanical and Aerospace Engineering, \\ University of Florida, \\ Gainesville, FL 32611 \\ e-mail: subhash@ufl.edu
}

Tensile and compressive stress-strain responses were obtained for various densities of polymer foams. These experimental data were used to determine relevant engineering parameters (such as elastic moduli in tension and compression, ultimate tensile strength, etc.) as a function of foam density. A phenomenological model applicable for both compressive and tensile responses of polymeric foams is validated by comparing the model to the experimentally obtained compression and tensile responses. The model parameters were analyzed to determine the effect of each parameter on the mechanical response of the foam. The engineering parameters were later compared to the appropriate model parameters and a good correlation was obtained. It was shown that the model indeed captures the entire compressive and tensile response of polymeric foams effectively. [DOI: $10.1115 / 1.3026556]$

\section{Introduction}

Cellular materials have been used in many automotive, aerospace, and military structures due to their light weight and ease of manufacture. Their low density and capability to absorb energy efficiently make them ideal candidates for passenger or cargo protection. Cellular materials are also used for noise reduction because of their ability to provide vibrational damping. For all these reasons the mechanical behavior of cellular structures continues to be pursued through experimental, numerical, and analytical approaches. Numerous experimental studies have been undertaken to investigate tensile [1-4], compressive [2,4], shear [4], and fracture $[3,5]$ properties of metallic and polymeric foams. New engineering parameters such as "energy efficiency" $(\eta)$ and "ideality" (I) were introduced to demonstrate the effectiveness of foams to cushion impact loading [6]. Recently, nonconventional specimens such as trapezoidal shapes [7] were used to explore the effects of specimen size on mechanical response.

Numerous concurrent analytical modeling efforts have also been undertaken to investigate the behavior of cellular materials. Rusch $[8,9]$ proposed one of the earlier models that is purely empirical where the stress and strain were related through the elastic modulus, and a mapping function whose parameters were determined experimentally. While such a stress-strain relationship could capture the compressive response of a polymeric foam, the parameters in the function bear no physical meaning. Several other models with similar features have been proposed [10-12]. A summary of analytical models for foam behavior are available in Ref. [13]. The most common material property that can be related to the behavior of cellular materials is the relative density $(\phi)$, which is defined as the ratio of the foam density to the density of the solid phase. The commonly used relation is of the form $X_{f}$ $=X_{s} \phi^{n}$ [14] where $X_{f}$ and $X_{s}$ represent mechanical properties of the foam and the solid material, respectively. The exponent $n$ may be obtained empirically or can be found through micromechanical models. Gibson and Ashby [15] used the above power-law func-

\footnotetext{
${ }^{1}$ Corresponding author.

Contributed by the Materials Division of ASME for publication in the JOURNAL OF Engineering Materials and Technology. Manuscript received January 25, 2008; final manuscript received July 1, 2008; published online December 18, 2008. Assoc. Editor: Thomas Siegmund.
}

tion to model material parameters and to map the stress-strain behavior for a number of cellular materials. Their analysis reaffirmed that material parameters pertaining to the mechanical response are highly dependent on the relative density and the mechanical properties of the solid phase of the foam. They also explored the effects of cell geometry and found that the response of foam differed between open and closed cell structures.

While the analytical models have focused on capturing the overall stress-strain behavior, more intricate and complex modeling efforts that focus on the influence of microstructural features (e.g., cell size, cell wall thickness, irregularities in the cell architecture, etc.) were studied by numerical methods [16-19]. Many of the above models, both analytical and numerical, are suitable for either compressive or tensile responses but not for both.

Liu and Subhash (LS) [13] proposed a six parameter phenomenological model that can capture the entire compressive stressstrain response of the foam, i.e., the initial elastic response, plateau region, and densification phase. The model has been shown to capture the monotonic loading response of many different types of foam, including elastomeric and crushable polymer foams. Since the response of metallic foams is very similar to polymeric foams [15] it is theorized that the model can also capture their behavior. Through extensive experimentation on several epoxy foams $[20,21]$ with different relative densities, they developed functional forms for each of the model parameters and developed crushability maps, which can assist in the identification of the most suitable initial foam density for maximum energy absorption. Most recently, Avalle et al., [22] proposed a similar model. Modifications to the LS model were further provided by Liu and O'Toole [23] by incorporating additional terms to capture the small softening-like behavior following the initial collapse and the response just before the onset of densification. While this model demonstrates the ability to capture small details of the foam response, the added complication in determining the additional parameters makes implementation of the above model less attractive.

The stress-strain response of porous polymeric materials subjected to compressive loads has three distinct zones: (i) an elastic region, (ii) a plateau region, and (iii) a densification region. These regions along with the LS model fit (to be discussed later) are shown in Fig. 1. The plateau region (beyond the elastic response) is associated with the collapse of the cellular structure, which 


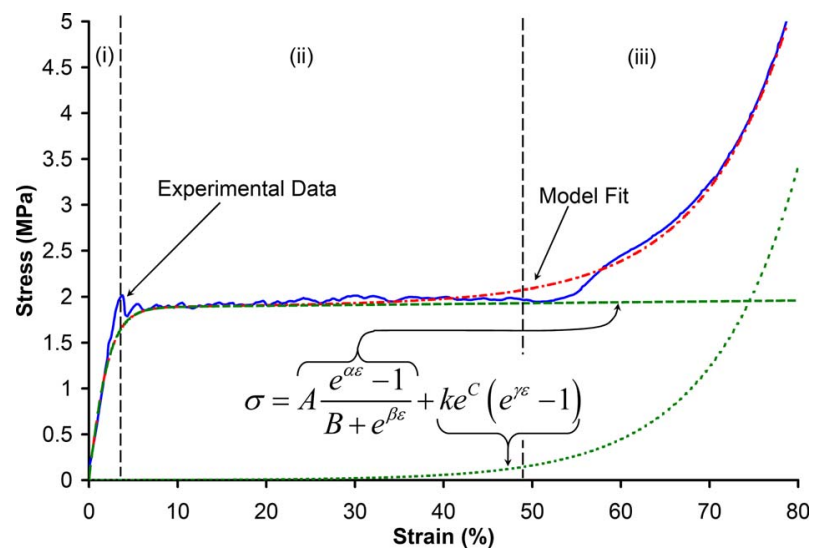

Fig. 1 Typical compressive stress-strain response of a polymer foam. (i) Elastic region, (ii) collapse region, and (iii) densification region. Note the effect of each term in the LS model used to fit the data.

gives the cellular material its energy absorption ability. The total compressive strain can be on the order of 40-80\% [15]. On the other hand, the tensile response may be limited to less than $10 \%$ [24]. The typical sequence of mechanisms that contributes to tensile strain are rotation and alignment of cell walls along the loading direction, elastic stretching of cell walls followed by their yield, and finally, crack propagation perpendicular to the loading axis across the entire specimen [14,15]. Although numerous models for compressive response are available, few models have been shown to capture both the tensile and compressive responses. In this paper we utilize the previously stated LS model, which has been shown to capture the compressive response [13,20,21,25], to validate its applicability to the tensile regime by making use of the combined tensile and compressive stress-strain responses of polymer foams of different densities.

\section{Experimental Procedure}

Five commercially available Divinycell ${ }^{\circledR}$ H-type foam sheets $25.4 \mathrm{~mm}$ in thickness with nominal densities ranging from $45 \mathrm{~kg} / \mathrm{m}^{3}$ to $200 \mathrm{~kg} / \mathrm{m}^{3}$ were obtained from DIAB group (DeSoto, TX). Divinycell ${ }^{\circledR}$ is a cross linked polyvinyl chloride (PVC) thermoplastic closed cell foam. Optical images of select densities are shown in Fig. 2. Cylindrical specimens $25.4 \mathrm{~mm}$ in diameter were cut from each sheet, and the apparent density of multiple specimens were measured using a gas pycnometer [26]. This device also provides the percentage of open cell volume for each specimen. These values are provided in Table 1 . All mechanical tests were performed at room temperature $\left(23^{\circ} \mathrm{C}\right)$ and at a rate of $2.54 \mathrm{~mm} / \mathrm{min}$ using either a MTS ${ }^{\circledR}$ (Eden Prairie, MN) or Tinius-Olsen ${ }^{\circledR}$ (Horsham, PA) universal testing machine (UTM). For compression testing, each specimen was marked with a grid of horizontal lines to track the uniformity of deformation during the test $[13,20,21]$. Upon loading it was noted that the foam specimens deformed unevenly along the vertical surfaces, as shown in Fig. 3. This behavior is due to premature collapse of the

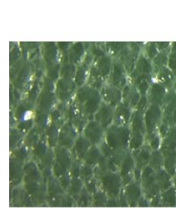

H60

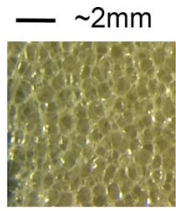

H130

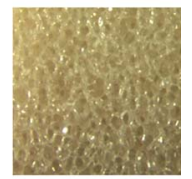

$\mathrm{H} 200$
Fig. 2 Optical images of the cellular structure of Divinycell ${ }^{\circledR}$ foam of different densities
Table 1 Nominal and measured densities for Divinycell ${ }^{\circledR}$ foam

\begin{tabular}{cccc}
\hline \hline \multirow{2}{*}{$\begin{array}{c}\text { Divinycell } \\
\text { name }\end{array}$} & Nominal & Measured & $\begin{array}{c}\text { Closed cell } \\
\text { percentage }\end{array}$ \\
\cline { 2 - 3 } & 45 & 58.70 & 89.60 \\
H45 & 60 & 72.27 & 93.43 \\
H60 & 100 & 106.40 & 94.27 \\
H100 & 130 & 139.40 & 94.77 \\
H130 & 200 & 223.57 & 97.25 \\
H200 & & & \\
\hline \hline
\end{tabular}

damaged cells that lie along the outer surface. The collapse then leads to instabilities along the surface resulting in buckling of the entire specimen. To avoid this mode of failure it was decided to test the specimens in a transparent confinement cell (shown in Fig. 4). Comparison of the compressive behavior of specimens with and without confinement is also presented in Fig. 3. It is clear that the confinement has little effect on the compressive stress-strain response.

Specimens for tensile testing were prepared by using a modified procedure based on ASTM D1623-3 [27]. The cylindrical foam specimens were bonded between two aluminum tabs of equal diameter, as shown in Fig. 5. These tabs allow a specimen to be

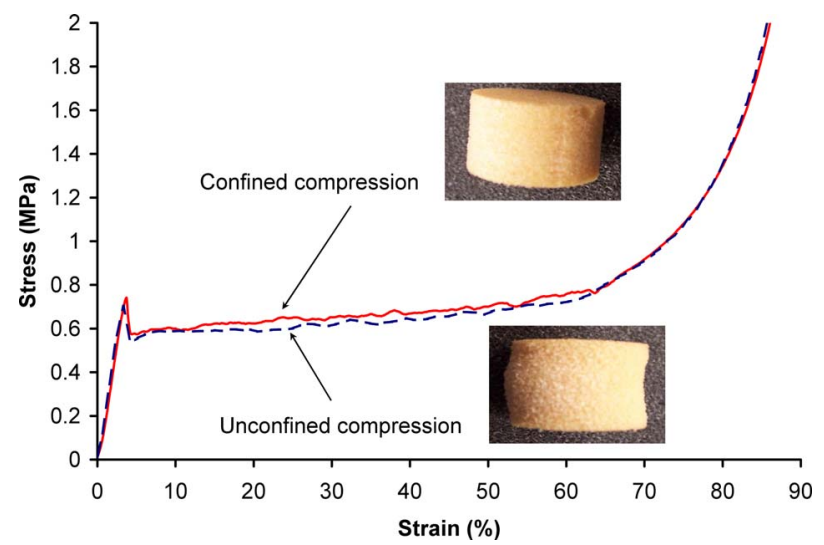

Fig. 3 Comparison between confined and unconfined compression of Divinycell ${ }^{\circledR} \mathrm{H} 45$ foam

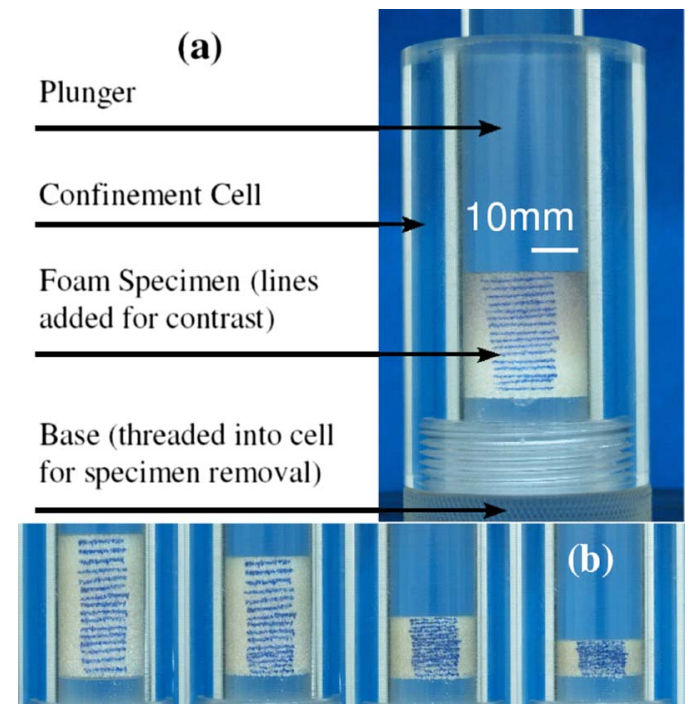

Fig. 4 (a) Test fixture for confined compression of cellular specimen and $(b)$ snapshots of a deformed specimen at selected intervals 


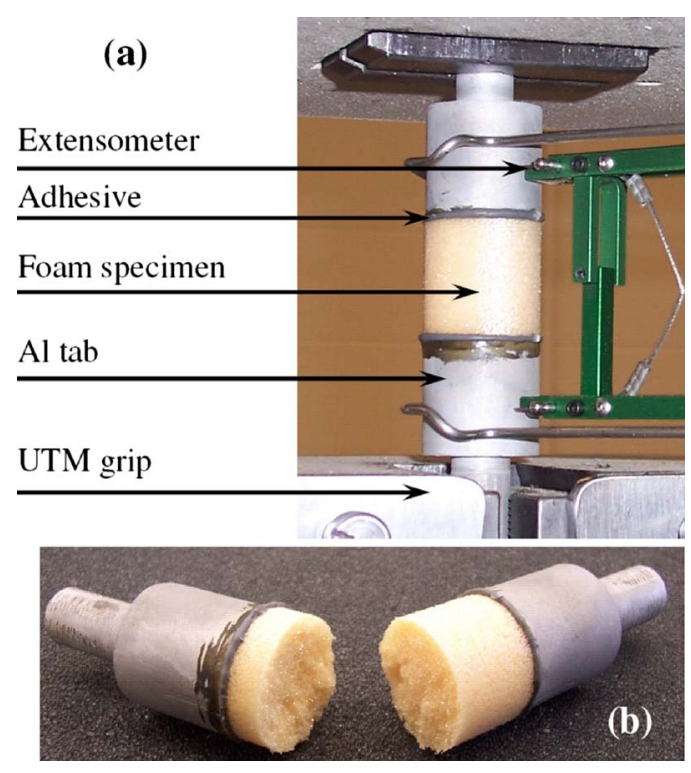

Fig. 5 (a) Tensile test fixture in UTM with test specimen bonded between two aluminum tabs, and $(b)$ fractured foam specimen

gripped and subjected to tensile loading. Aluminum was chosen as the fixture (tab) material due to its high stiffness relative to the foam specimen. Three adhesives were tested for their bond strength between the aluminum tabs and a foam specimen; JB Weld $^{\circledR}\left(\right.$ JB Weld $^{\circledR}$, Sulphur Springs, TX), ProSet ${ }^{\circledR}$ epoxy (Gougeon Brothers, Bay City MI), and Depend ${ }^{\circledR} 220$ (Loctite ${ }^{\circledR}$, Rocky Hill, CT). Initial tensile experiments were conducted by bonding the two aluminum tabs so as to measure the strength of the adhesive joint. While each adhesive was shown to have sufficient strength (well above the tensile strength of the foam) with negligible tensile strain during the test, JB Weld was chosen due to its ease of application. To ensure a strong adhesive bond between the specimen and the fixture, the tab surface was machined and beadblasted to create a rough surface. The surface was then cleaned using a degreasing solvent (CSM-2), surface Conditioner A, and Neutralizer 5A produced by Vishay Micro-Measurements (Malvern, PA). A thin coating of adhesive was then applied to the prepared surfaces, which were then bonded to the two sides of the cylindrical specimen. Since the adhesive is very thin compared to the specimen size, any contribution of strain in the bond layer could be neglected. The prepared specimens were then loaded into a UTM and subjected to a tensile force until failure occurred. Displacement was measured using an extensometer, as shown in Fig. 5. For each density of foam five specimens were tested in compression and another five specimens in tension.

\section{Results}

The compressive and tensile engineering stress-strain responses (five each) of the five different densities of foam are presented in Fig. 6. For clarity the tensile regime is magnified. Note that the scatter in the response of five specimens at each density is not so significant. In the tensile regime the scatter appears to be large due to a difference in scale between compression and tensile responses. Clearly the behavior of the foam in tension differs considerably from that in compression, yet both show a strong dependency on density. The nominal (engineering) compressive strain can be as high as $90 \%$ whereas the tensile strain is less than $8 \%$. With increase in density, the initial slope, collapse stress, and plateau stress in compression, as well as the initial slope and fracture strength in tension, increase gradually. The elastic portions of the stress-strain curves in both tension and compression are nonlinear and therefore, their slopes were incrementally cal-

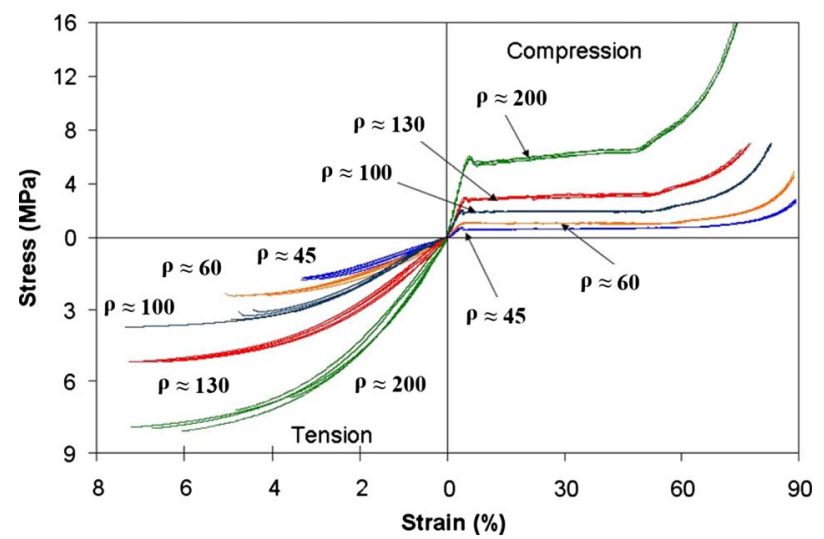

Fig. 6 Tensile and compressive responses of Divinycell ${ }^{\circledR}$ PVC foam specimens of different densities. Note the change in scale for the tension response.

culated over the entire elastic range and an average value was chosen. In most of the compression test results there is a small drop in stress at the end of the elastic region. The peak stress just before the plateau region is defined as the "collapse stresses." The plateau region represents the collapse behavior of the cellular material until the densification region is reached. After the entire collapse of the cellular structure, the specimen is fully densified at which point its behavior is similar to the compressive response of the solid material. In this sense, the foam specimens never truly fail as they can still maintain a compressive load. In contrast, the specimens subjected to tensile loading fractured into two pieces at relatively low strains, as shown in Fig. 5(b). The strain at the onset of densification $\left(\varepsilon_{\text {dens }}\right)$ is sometimes defined as a "deformation limit" for the analysis of the energy absorption characteristics of the foam [28]. Beyond this limit the usefulness (or efficiency) of the foam to absorb energy is limited. It was shown by Li et al., [29] that by plotting the energy efficiency parameter $(\eta)$ against strain, the strain at the onset of densification may be determined as the strain associated with peak efficiency. There is a noticeable difference between the measured compressive and tensile elastic moduli. It is common for polymer foams to exhibit this phenomenon where the transition between tensile and compressive regions appears to be discontinuous. This is associated with the nonlinear nature of the elastic responses in tension and compression [30].

Engineering parameters extracted from the above plots are summarized in Table 2 . The compression modulus $\left(E_{c}\right)$ and the collapse stress $\left(\sigma_{c}\right)$ values determined from the above stress-strain responses are plotted against the foam density in Fig. 7. Both modulus and collapse stress increase with density of foam. Similarly, the values for tensile modulus $\left(E_{t}\right)$, and ultimate tensile strength $\left(\sigma_{\text {ult }}\right)$, plotted in Fig. 8, increase linearly with density. Superposed on both the above plot are the trend lines for the data.

Table 2 Engineering parameters from tension and compression testing of Divinycell ${ }^{\circledR}$ foam

\begin{tabular}{ccccccc}
\hline \hline & \multicolumn{3}{c}{ Tension } & \multicolumn{3}{c}{ Compression } \\
\cline { 2 - 7 } \begin{tabular}{c} 
Density $\mathrm{kg} / \mathrm{m}^{3}$ \\
\cline { 2 - 7 }
\end{tabular} & $\sigma_{\text {ult }}(\mathrm{MPa})$ & $\varepsilon_{\text {ult }} \%$ & $E_{t}(\mathrm{MPa})$ & $\sigma_{c}(\mathrm{MPa})$ & $E_{c}(\mathrm{MPa})$ & $\varepsilon_{\text {dens }}(\%)$ \\
\hline 45 & 1.71 & 3.23 & 68.10 & 0.74 & 24.64 & 64.44 \\
60 & 2.28 & 4.16 & 74.63 & 1.10 & 35.54 & 62.94 \\
100 & 3.39 & 5.29 & 112.72 & 2.02 & 67.73 & 58.9 \\
130 & 5.19 & 6.67 & 147.60 & 2.96 & 87.63 & 58.22 \\
200 & 7.58 & 5.70 & 226.36 & 5.97 & 145.06 & 54.86 \\
\hline \hline
\end{tabular}




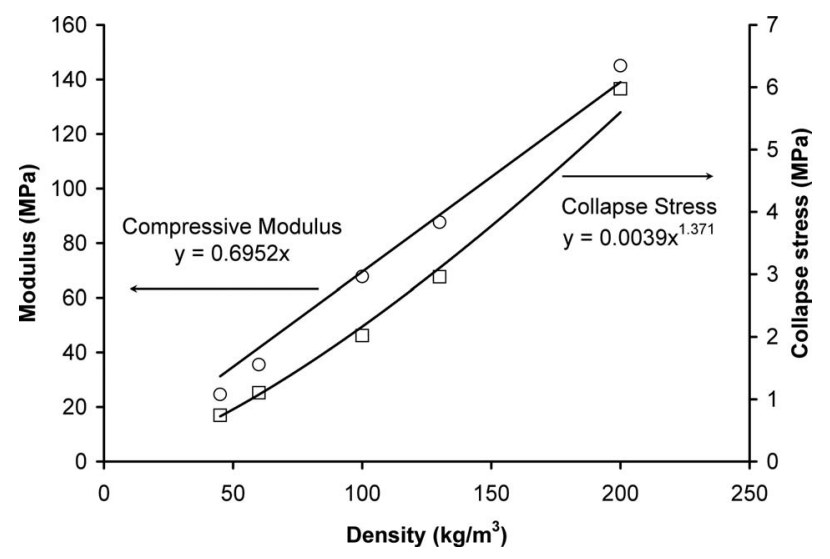

Fig. 7 Trends in compression modulus and collapse stress as a function of foam density

\section{Model Validation}

The model proposed by Liu and Subhash [13] is used in this work to capture the entire tensile-compression behavior of polymer foams. To accomplish this task the experimental results for uniaxial tension and compression for each density of foam were combined to create a set of data that may be fit using the LS model. The model consists of two terms incorporating the six parameters shown below

$$
\sigma=A \frac{e^{\alpha \varepsilon}-1}{B+e^{\beta \varepsilon}}+k e^{C}\left(e^{\gamma \varepsilon}-1\right)
$$

The first term in the model captures the elastic response and the plateau region, while the second term controls the densification behavior and is only effective in the compression regime. This behavior was shown in Fig. 1. For consistency in units on the right hand side, a pseudoparameter $k$ has been introduced, which has units of stress and a value of unity. Each of the six parameters $(A$, $B, C, \alpha, \beta$, and $\gamma)$ can be shown to have a specific role in mapping the stress-strain response of the foam. It has been shown that the slope of the plateau region is controlled by the $\alpha$ and $\beta$ terms $[13,21]$. For $\alpha>\beta$ a hardeninglike response and for $\alpha<\beta$ a softeninglike behavior are achieved. The constant stress plateau region can be captured by setting $\alpha=\beta$. Assuming a perfectlyplastic plateau region $(\alpha=\beta)$ the first term reduces to $A$ for large compressive strains $(\varepsilon \rightarrow \infty)$ and to $-\frac{A}{B}$ for large tensile strains $(\varepsilon \rightarrow-\infty)$. This indicates that the parameter $A$ represents the plateau or collapse stress of the material, i.e.,

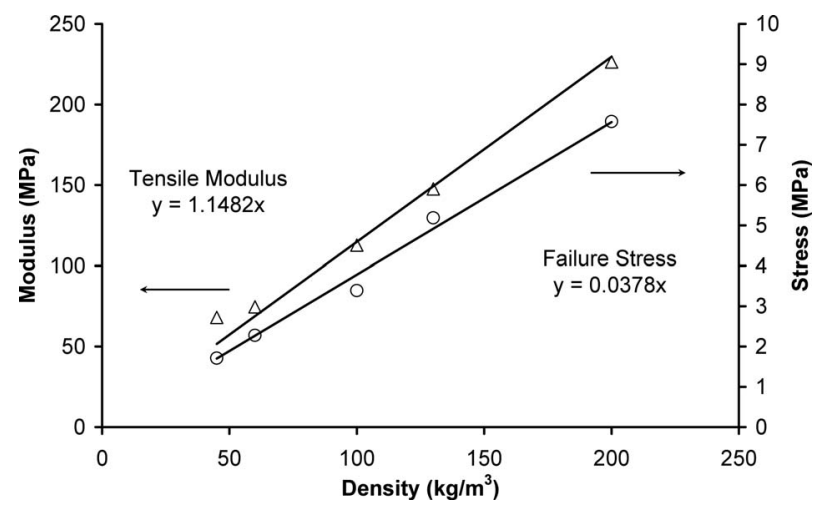

Fig. 8 Trends in the measured tensile engineering parameters as a function of foam density

$$
A \approx \sigma_{c}
$$

The parameter $B$ represents the ratio of collapse stress to ultimate tensile stress, i.e.,

$$
B \approx \frac{\sigma_{c}}{\sigma_{\mathrm{ult}}} \text { or } \sigma_{\mathrm{ult}} \approx \frac{A}{B}
$$

The elastic modulus of the material may be found by differentiating the first term and letting $\varepsilon$ approach zero, to obtain

$$
E=\frac{A \alpha}{1+B}
$$

Since the parameters $A$ and $B$ are now defined in terms of engineering parameters, we see that the elastic modulus is controlled by only the parameter $\alpha$. Substituting Eqs. (2) and (3) into Eq. (4) we obtain

$$
E \approx \frac{\sigma_{c} \alpha}{1+\sigma_{c} / \sigma_{\mathrm{ult}}}
$$

We can now define $\beta$ as the model parameter controlling the behavior of the plateau region, as mentioned above.

The parameters $C$ and $\gamma$ control the densification region $[13,20,21]$. Parameter $C$ controls the onset of densification while the rate of densification remains constant, and parameter $\gamma$ controls the rate of densification while the onset of densification (parameter $C$ ) is kept constant. Although each parameter has been shown to control a separate phase of response in the behavior of the foam there are some interdependencies between parameters. For example the slope of the collapse region is dependent on the difference between $\alpha$ and $\beta$, and therefore as $\alpha$ increases so does the parameter $\beta$ and vice versa. However some interdependency is necessary for this phenomenological model to capture the complex nonlinear response of these foams.

Having experimentally determined the compressive and tensile stress-strain responses of five foams with different densities (Fig. 6), we will now embark on fitting the LS model to the entire compression and tensile regimes. Because foams are mostly used in compression for energy absorption, we consider the compression response to be positive and the tensile response to be negative. Also, in the following analysis the strain has units of percentage strain. Recall that we have conducted five tensile and five compression tests for each density of foam. Obviously there is some statistical variation in all these responses. To account for this variation we have considered all the possible combinations of tensile and compression responses thus creating 25 possible curves spanning both tension and compression for each foam density. The LS model was then applied to each of these 25 data sets using a MATLAB ${ }^{\circledR}$ code including the Statistical Toolbox function "nlinfit," and from each curve the six model parameters were determined. An average value for each parameter was then determined from the 25 sets of data for each foam density. These average model parameters are presented in Table 3. From these average model parameters the stress-strain curves were reconstructed and then compared to an experimentally obtained curve, as shown in Fig. 9. Clearly the model is very effective in capturing most of the compressive response, as well as the tensile response. The model fits the elastic response of the tensile data, as well as captures the plateau and densification regions of the compression data. It fails to capture the elastic portion of the compressive response due to a discontinuity in the elastic responses of the compression and tension curves (see $E_{t}$ and $E_{c}$ values in Table 2) at the origin. This discontinuity is caused by the difference in the two elastic moduli in tension and compression. However, since the elastic region in compression is very small $(<5 \%)$ when compared to the large $(>65 \%)$ plateau region, this deviation can be considered not so significant. Also since the tensile strains are much smaller then the compressive strains it is more critical to capture the elastic region of the tensile response. Thus, it can be seen that the model indeed 
Table 3 LS model parameter averages for Divinycell ${ }^{\circledR}$ foams

\begin{tabular}{ccccccccccc}
\hline \hline Parameter & $\mathrm{H} 45$ & $\pm \mathrm{SD}$ & $\mathrm{H} 60$ & $\pm \mathrm{SD}$ & $\mathrm{H} 100$ & $\pm \mathrm{SD}$ & $\mathrm{H} 130$ & $\pm \mathrm{SD}$ & $\mathrm{H} 200$ & $\pm \mathrm{SD}$ \\
\hline$A$ & 0.58 & \pm 0.014 & 1.06 & \pm 0.017 & 1.92 & \pm 0.031 & 2.80 & \pm 0.042 & 5.25 & \pm 0.166 \\
$B$ & 0.30 & \pm 0.010 & 0.45 & \pm 0.030 & 0.50 & \pm 0.018 & 0.54 & \pm 0.009 & 0.65 & \pm 0.024 \\
$C$ & -10.96 & \pm 0.196 & -9.09 & \pm 0.203 & -6.92 & \pm 0.289 & -6.86 & \pm 0.670 & -6.14 & \pm 0.170 \\
$\alpha$ & 1.09 & \pm 0.097 & 1.01 & \pm 0.119 & 0.71 & \pm 0.032 & 0.75 & \pm 0.050 & 0.74 & \pm 0.077 \\
$\beta$ & 1.09 & \pm 0.097 & 1.01 & \pm 0.119 & 0.71 & \pm 0.031 & 0.75 & \pm 0.050 & 0.74 & \pm 0.077 \\
$\gamma$ & 0.13 & \pm 0.002 & 0.12 & \pm 0.002 & 0.10 & \pm 0.003 & 0.11 & \pm 0.008 & 0.11 & \pm 0.002 \\
\hline \hline
\end{tabular}

captures the entire tensile and compressive responses reasonably well for all the foam densities considered in this study.

\section{Analysis of Model Parameters}

The trends in the model parameters $A, B, C, \alpha, \beta$, and $\gamma$ are now plotted as a function of density in Fig. 10. It is noted that the parameters $A, B$, and $C$ increase with density whereas the parameters $\alpha$ and $\beta$ decrease with density. While the preceding five parameters show a strong dependency on density, parameter $\gamma$ remains relatively constant. Recall that the parameter $\gamma$ relates to rate of densification after the complete collapse of the cellular structure and hence should strongly correlate to the response of the solid phase of the foam. Since all the foam specimens are made from the same solid phase (PVC), this parameter should remain relatively constant for all the foam densities, which is well captured by the model results.

Finally, we will compare the model parameters $A, B$, and $\alpha$ (Eqs. (2)-(4)) to the appropriate engineering parameters given in Table 2. Again, recall that the value of $E$ determined by the model
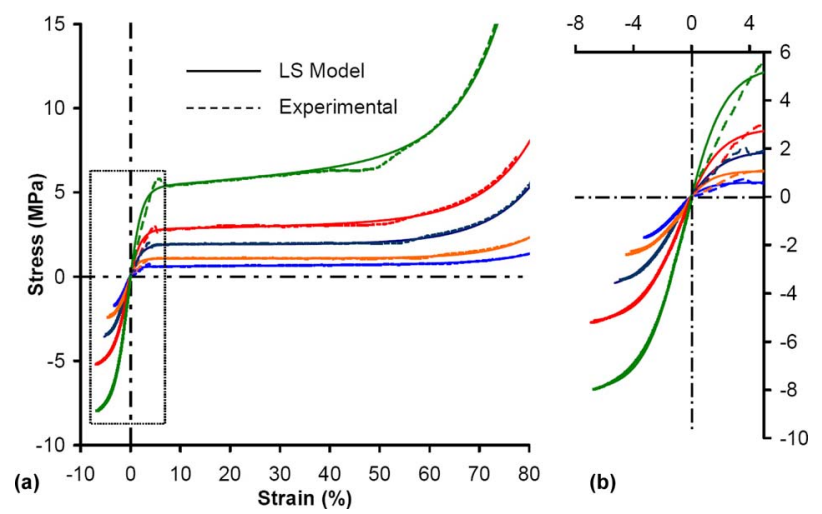

(b)

Fig. 9 (a) LS model fit using average parameter values and a single representative test data, and (b) an expanded view of the model fit in the elastic region

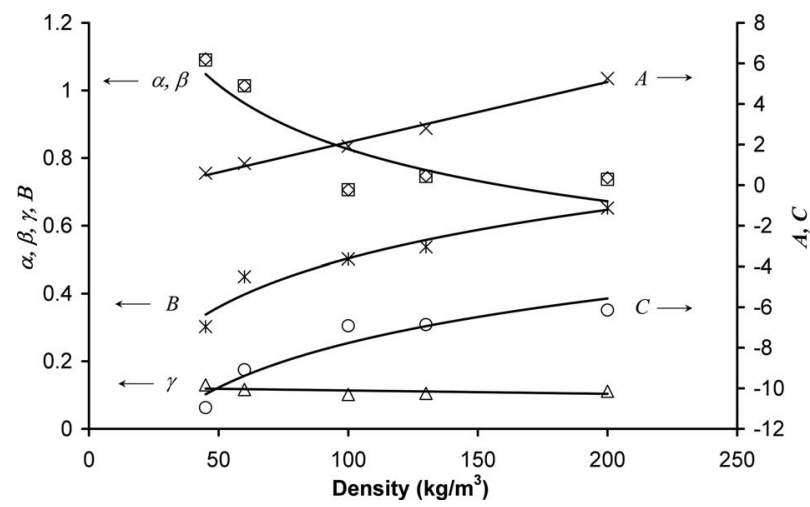

Fig. 10 Trends in the LS model parameters as functions of foam density parameters (Eq. (4)) was obtained by setting $\varepsilon=0$ in Eq. (1). Since zero stress and strain are defined for both tension and compression, this modulus in Eq. (4) cannot be distinguished as one or the other but should represent both. However, the model fits the tensile elastic portion much more effectively than the compressive elastic response (see Fig. 9). Therefore the value of $E$ determined from model parameters should correlate to the measured tensile elastic modulus $\left(E_{t}\right)$ much better then the compressive modulus $\left(E_{c}\right)$. The model parameter relations (Eqs. (2)-(4)), as well as the corresponding engineering parameters (Table 2), are plotted against density in Fig. 11 along with the best fit lines. Clearly, the agreement between the two is quite satisfactory. By grouping the model parameters in this way to form engineering parameters, it is shown that the LS model captures the behavior of polymeric foam reasonably well. This procedure also demonstrates the ability of the LS model to map the stress-strain curve using engineering parameters.

\section{Discussion}

The proposed model is purely phenomenological and hence the parameters can be chosen to capture any response irrespective of the loading type (compression or tension). In this particular case, the model is designed to capture both the elastic as well as the highly nonlinear inelastic response of cellular materials in both tensile and compressive regimes. The model may also be extended to capture other responses including, for example, the torsional behavior. If results from the tensile, compressive, and shear properties were then combined with an appropriate failure envelope, the multiaxial behavior could then be predicted using the model. There are several such envelopes proposed in literature based on uniaxial and other combined loading tests $[4,15]$.

As mentioned above, the behavior of a foam material is dependant on both the density as well as the mechanical properties of the solid material. To effectively capture the foam behavior using

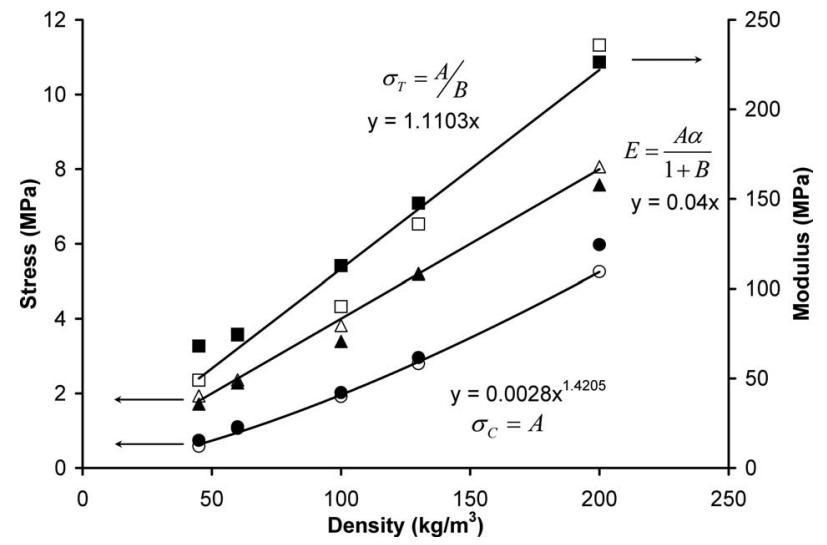

Fig. 11 Grouped LS model parameters representing common engineering parameters versus foam density. Note the trends are similar to those seen in curves of engineering parameters versus foam density. Open symbols represent model parameters and filled symbols represent experimental values. 
this method several different types of foam must be tested. If tests were performed on several types of foam with different densities it would be possible to form functional relations between the LS model parameters and both density and the solid material properties.

The LS model is not without limitations. First, the model is suitable only for monotonic loading. The unloading in the model is purely elastic such that it follows the same curve during loading and unloading. Second, the tensile failure strain is not captured by the model. However, the "failure strain" may be prescribed in a numerical simulation to indicate the onset of failure. Thus, one needs to have experimental data to prescribe this critical strain. Finally, the model suggests that the elastic modulus is dependent on the collapse stress and the ultimate tensile stress (Eqs. (4) and (5)). However these limitations are not uncommon phenomenological models. The benefit of using the LS model is that it can capture the entire tensile and compressive responses all the way to densification (i.e., up to $70 \%$ strain) using a single equation. Such capabilities are helpful in modeling the behavior of realistic structures under multiaxial loading where large deformations are expected. Examples of such scenarios include determination of the crash box (filled with foam) behavior during an automobile collision.

\section{Conclusions}

The applicability of the LS model to the entire compressiontension constitutive response of polymeric foams has been shown using experimental data generated from five different densities of Divinycell $^{\circledR}$ foam. This data was also used to determine the compressive and tensile elastic moduli, collapse stress, failure strength, and failure strain, which were all shown to increase with foam density. The densification strain was also determined from experimental data. Model parameters were shown to be related to the engineering properties of the foams.

\section{Acknowledgment}

The authors would like to gratefully acknowledge the support of Ms. Sara Trofka, DIAB Inc., DeSoto, TX for providing foam panels used in this study.

\section{References}

[1] Kraatz, A., Moneke, M., and Kolupaev, V., 2006, "Long-Term Tensile and Compressive Behavior of Polymer Foams," J. Cell. Plast., 42(3), pp. 221-228.

[2] Goods, S. H., Neuschwanger, C. L., Henderson, C. C., and Skala, D. M., 1998, "Mechanical Properties of Crete, a Polyurethane Foam," J. Appl. Polym. Sci., 68(7), pp. 1045-1055.

[3] Kabir, M. E., Saha, M. C., and Jeelani, S., 2006, "Tensile and Fracture Behavior of Polymer Foams," Mater. Sci. Eng., A, 429(1-2), pp. 225-235.

[4] Gdoutos, E. E., Daniel, I. M., and Wang, K. A., 2001, "Failure of Cellular Foams Under Multiaxial Loading," Composites, Part A, 33(2), pp. 163-176.

[5] Bazant, Z. P., Yong, Z., Goangseup, Z., and Daniel, I. M., 2003, "Size Effect and Asymptotic Matching Analysis of Fracture of Closed-Cell Polymeric Foam,” Int. J. Solids Struct., 40(25), pp. 7197-7217.

[6] Miltz, J., and Gruenbaum, G., 1981, "Evaluation of Cushioning Properties of
Plastic Foams From Compressive Measurements," Polym. Eng. Sci., 21(15), pp. $1010-1014$.

[7] Wierzbicki, T., and Doyoyo, M., 2003, "Determination of the Local StressStrain Response of Foams," ASME J. Appl. Mech., 70(2), pp. 204-211.

[8] Rusch, K. C., 1970, "Load-Compression Behavior of Brittle Foams," J. Appl. Polym. Sci., 14(5), pp. 1263-1276.

[9] Rusch, K. C., 1969, "Load-Compression Behavior of Flexible Foams," J. Appl. Polym. Sci., 13(11), pp. 2297-2311.

[10] Meinecke, E. A., and Schwaber, D. M., 1970, "Energy Absorption in Polymeric Foams," J. Appl. Polym. Sci., 14(9), pp. 2239-2248.

[11] Nagy, A., Ko, W. L., and Lindholm, U. S., 1974, "Mechanical Behavior of Foamed Materials Under Dynamic Compression," J. Cell. Plast., 10(3), pp. $127-134$.

[12] Sherwood, J. A., and Frost, C. C., 1992, "Constitutive Modeling and Simulation of Energy Absorbing Polyurethane Foam Under Impact Loading," Polym. Eng. Sci., 32(16), pp. 1138-1146.

[13] Liu, Q., and Subhash, G., 2004, "A Phenomenological Constitutive Model for Foams Under Large Deformations," Polym. Eng. Sci., 44(3), pp. 463-473.

[14] Hilyard, N. C., and Cunningham, A., 1994, Low Density Cellular Plastics. Physical Basis of Behaviour, N. C. Hilyard and A. Cunningham, eds., Chapman and Hall, New York.

[15] Gibson, L. J., and Ashby, M. F., 1999, Cellular Solids: Structure and Properties, Cambridge University Press, Cambridge.

[16] Gao, X. L., Li, K., and Subhash, G., 2006, "Effects of Cell Shape and Strut Cross-Sectional Area Variations on the Elastic Properties of ThreeDimensional Open-Cell Foams," J. Mech. Phys. Solids, 54(4), pp. 783-806.

[17] Li, K., Gao, X. L., and Subhash, G., 2005, "Effects of Cell Shape and Cell Wall Thickness Variations on the Elastic Properties of Two-Dimensional Cellular Solids,” Int. J. Solids Struct., 42(5-6), pp. 1777-1795.

[18] Gong, L., and Kyriakides, S., 2005, "Compressive Response of Open Cell Foams. Part II: Initiation and Evolution of Crushing," Int. J. Solids Struct., 42(5-6), pp. 1381-1399.

[19] Gong, L., Kyriakides, S., and Jang, W. Y., 2005, "Compressive Response of Open-Cell Foams. Part I: Morphology and Elastic Properties," Int. J. Solids Struct., 42(5-6), pp. 1355-1379.

[20] Subhash, G., and Liu, Q., 2004, "Crushability Maps for Structural Polymeric Foams in Uniaxial Loading Under Rigid Confinement,” Exp. Mech., 44(3), pp. 289-294.

[21] Liu, Q., Subhash, G., and Gao, X.-L., 2005, “A Parametric Study on Crushability of Open-Cell Structural Polymeric Foams," J. Porous Mater., 12(3), pp. 233-248.

[22] Avalle, M., Belingardi, G., and Ibba, A., 2007, "Mechanical Models of Cellular Solids: Parameters Identification From Experimental Tests," Int. J. Impact Eng., 34(1), pp. 3-27.

[23] Liu, Q., and O'Toole, B., 2007, "Behavior Pattern and Parametric Characterization for Low Density Crushable Foams," J. Mater. Process. Technol., 191(1-3), pp. 73-76.

[24] Throne, J. L., 1982, "Structural Foams," Mechanics of Cellular Plastics, N. C. Hilyard, ed., Macmillan, New York, pp. 236-322.

[25] Subhash, G., Liu, Q., and Gao, X.-L., 2006, "Quasistatic and High Strain Rate Uniaxial Compressive Response of Polymeric Structural Foams," Int. J. Impact Eng., 32(7), pp. 1113-1126.

[26] ASTM Standard D1621-00, "Standard Test Method for Compressive Properties of Rigid Cellular Plastics," ASTM International, West Conshohocken, PA, www.astm.org.

[27] ASTM Standard C273-00, "Standard Test Method for Shear Properties of Sandwich Core Materials," ASTM International, West Conshohocken, PA, www.astm.org.

[28] Ashby, M. F., 2000, Metal Foams: A Design Guide, Butterworth-Heinemann, Boston.

[29] Li, Q. M., Magkiriadis, I., and Harrigan, J. J., 2006, "Compressive Strain at the Onset of Densification of Cellular Solids," J. Cell. Plast., 42(5), pp. 371-392.

[30] Menges, G., and Knipschild, F., 1982, "Stiffness and Strength-Rigid Plastic Foams," Mechanics of Cellular Plastics, N. C. Hilyard, ed., Macmillan, New York, p. 401. 